\title{
Collision of One-Dimensional Nonlinear Chains
}

\author{
Shin-ichiro Nagahiro and Yoshinori Hayakawa \\ Department of Physics, Tohoku University, Sendai, Japan
}

(Dated: November 21, 2018)

\begin{abstract}
We investigate one-dimensional collisions of unharmonic chains and a rigid wall. We find that the coefficient of restitution (COR) is strongly dependent on the velocity of colliding chains and has a minimum value at a certain velocity. The relationship between COR and collision velocity is derived for low-velocity collisions using perturbation methods. We found that the velocity dependence is characterized by the exponent of the lowest unharmonic term of interparticle potential energy.

PACS numbers: 45.50.Tn, 05.45.-a, 46.40.-f
\end{abstract}

\section{INTRODUCTION}

In collisions between two bodies which have internal degrees of freedom, some of the initial translational energy is transformed into internal energy of the two bodies. This is the major cause of energy dissipation. To characterize the macroscopic features, a phenomenological parameter, the coefficient of restitution (COR),

$$
\eta=\frac{K_{r}}{K_{i}}
$$

where $K_{i}$ and $K_{r}$ are translational kinetic energy before and after the collision, respectively, is commonly used. Recent studies of collisions are mainly focused on the determination of $\eta$ from microscopic mechanisms.

Hertz developed the theory of collision between frictionless elastic bodies [1, 2] based on his static theory of elastic contact [3]. In the theory, it is assumed that in low-velocity collisions, the deformation of colliding bodies is given by the static theory and the production of vibration is totally ignored. Hence, the theory gives no information on energy dissipation. Plastic deformation is one of the possible means of kinetic energy dissipation during collisions. Taking this into account, the energy dissipation rate $1-\eta$ is found to increase with collision velocity with a power law with the exponent $1 / 2$ 沎. Considering viscoelastic properties, the dissipation rate increases with the exponent $2 / 5$ [5, 6, 7]. These results were confirmed experimentally [8, 9, 10].

The results presented above are based on quasi-static approximation, hence it is expected that they are restricted to low-velocity collisions. We believe that more general results will be obtained through microscopic simulations 11, 12, 13].

Sugiyama and Sasaki [14] and Basile and Dumont 115] considered collisions between simple one-dimensional chains and a rigid wall. A chain is composed of $n$ identical point particles which interact with nearest-neighbor particles. If we choose linear force as the interaction between the particles, the COR is independent of collision velocity and approaches unity in the thermodynamic limit.

For collision between metallic bodies, plastic deformation usually occurs even if collision velocity is low. The deformation exceeds the elastic regime in which Hooke's

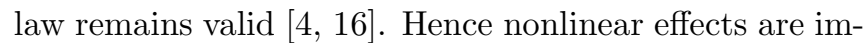
portant in realistic collisions. In this study, we choose nonlinear force as the interparticle interaction and perform the one-dimensional simulations of collision between a "nonlinear chain" and a rigid wall. We find that the COR of this collision has a minimum value at a certain velocity and derive the velocity dependence of the COR for low-velocity collisions using perturbation methods.

This paper is organized as follows. In the next section, we briefly review the studies of Sugiyama and Sasaki 14] and Basile and Dumont 15. In section 3, we present our results for collision between nonlinear chains and a wall. Finally, we summarize our results.

\section{COLLISIONS OF ONE-DIMENSIONAL HARMONIC CHAINS}

First we briefly discuss the collision of one-dimensional harmonic chains (model A) with a rigid wall as discussed by Sugiyama and Sasaki 14. Consider a chain composed of $n$ identical point particles labeled $j=1,2, \cdots n$. Each particle in the chain is linked to nearest-neighbor particles with a Hooke's spring, as illustrated in Fig.1. The Hamiltonian of this system is written as

$$
\mathcal{H}=\frac{m}{2} \sum_{j=1}^{n} \dot{x}_{j}^{2}+\frac{1}{2} m \omega^{2} \sum_{j=1}^{n-1}\left(x_{i+1}-x_{i}-\ell\right)^{2}+V_{w}
$$
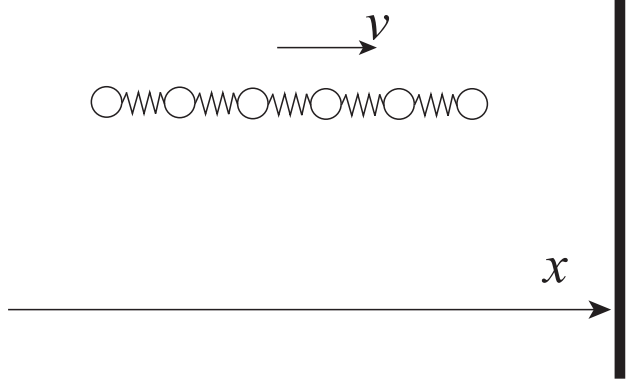

FIG. 1: Schematic of a one-dimensional chain and a rigid wall. 
where $x_{j}$ and $\dot{x}_{j}$ are the position and velocity of the $j$ th particle, respectively, and $\ell$ is the natural length of the springs. We assume the chain is homogeneous and all springs have the same spring constant $k\left(=m \omega^{2}\right) . V_{w}$ represents the hard-core potential of a rigid wall located at $x=0$ where collision takes place. During the collision process, only the particle $j=1$, which is at the end of the chain, interacts with the wall since the positional order of the particles is always kept as $x_{n}<x_{n-1}<\cdots<x_{2}<$ $x_{1}$. In the following discussion, we assume that the wall is so rigid that the particle $x_{1}$ simply reverses its velocity as $-\dot{x}_{1} \rightarrow \dot{x}_{1}$.

Although there is no dissipation term in the Hamiltonian, vibration energy which remains after the collision is regarded as the "dissipated" portion of energy. The $\mathrm{COR} \eta$ is therefore evaluated as

$$
\eta=1-\frac{E_{v i b .}}{E},
$$

where $E$ is the total energy and $E_{v i b}$. is the vibration energy which remains after the collision.

In the absence of $V_{w}$, the equation of motion is written as

$$
\ddot{\mathbf{x}}=-\omega^{2}(A \mathbf{x}+\mathbf{b}),
$$

where $\mathbf{x}=\left(x_{1}, x_{2}, \cdots, x_{n}\right), \mathbf{b}=(-\ell, 0, \cdots, 0, \ell)$ and $A$ is an $n \times n$ matrix of the form

$$
A=\left(\begin{array}{ccccccc}
1 & -1 & 0 & & & \cdots & 0 \\
-1 & 2 & -1 & 0 & & \cdots & 0 \\
0 & -1 & 2 & -1 & 0 & \cdots & 0 \\
\vdots & & & \ddots & & & \vdots \\
0 & & \cdots & & 0 & -1 & 1
\end{array}\right)
$$

Taking a principal-axis coordinate system in which $A$ is diagonal, internal vibration of the chain can be represented using $n$ noninteracting fundamental modes. In the presence of $V_{w}$, collision between the chain and the wall is realized as follows. Assume that the particle $j=1$ collides with the wall $f(n)$ times at $t_{1}, t_{2}, \cdots, t_{f(n)}$. The fundamental modes describe equi-energy elliptical orbits in phase space. The orbits are discontinuous at $t=t_{1}, t_{2}, \cdots, t_{f(n)}$.

For the following numerical simulations, we choose initial conditions

$$
\left\{\begin{array}{l}
x_{j}=\ell j \\
\dot{x}_{j}=-v_{0}(j=1,2, \cdots, n) .
\end{array}\right.
$$

Before collision, the chain has no internal vibration, i.e., zero temperature. Figure 2 shows the collision between the wall and the chain with $n=19$. Each line is a trace of the trajectory of a particle. In the plot, units on the time axis are taken as $\tau=(n-1) \ell / c_{l} . \tau$ indicates the duration in which the longitudinal sound wave propagates from one end of the chain to the other. Let us call the time $t_{c}$ during which the collision takes place "contact time". Here we note that contact time is almost equal to $2 \tau$

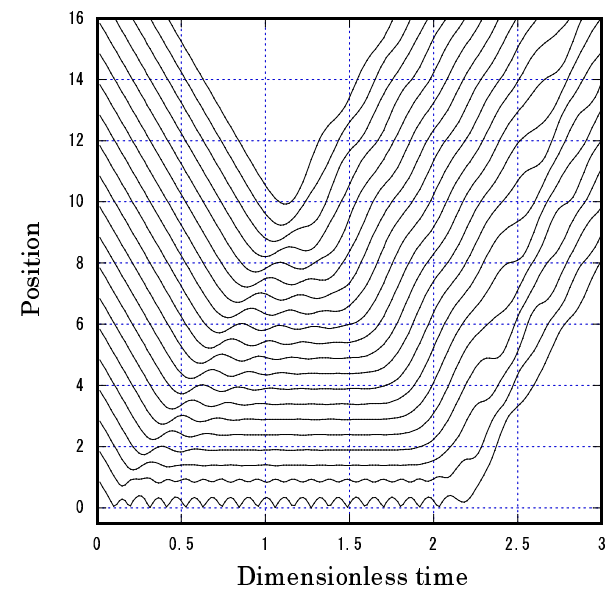

FIG. 2: Trajectories of the particles in the $n=20$ colliding chain. (Reduced units where $c_{l}=\tau=1$.)

for large $n$. COR and contact time are independent of the collision velocity of the chain because time intervals $\triangle t_{q}=t_{q+1}-t_{q}$ are independent of initial velocity for every $q$ and the orbits retain similar forms even if initial velocity of the chain varies. Therefore, $E_{j} / E$, the ratio of the $j$-th fundamental mode energy to total energy, does not depend on initial velocity. The COR, which is a ratio of energy, also does not depend on initial velocity. Hereafter, we use the reduced unit $c_{l}=\tau=1$, setting $\ell$, $m$ and $k$ as unity.

In model $\mathrm{A}$, the coefficient of restitution $\eta$ depends only on $n$. An approximate expression of $\eta$ was derived by Basile and Dumont 15. To simplify the problem, we assume that $\triangle t_{q}$ and the velocity before the $q$-th collision $v_{q}$ are constant for every $q$. Numerical simulations show that these are good approximations. Under this assumption, the vibration energy of the $j$-th mode after the collision process is given as

$$
E_{j}=\frac{4 m}{n} v^{2} \cos ^{2}\left(\frac{\pi j}{2 n}\right) \frac{\sin ^{2}\left\{\omega_{j} f(n) \triangle t / 2\right\}}{\sin ^{2}\left\{\omega_{j} \triangle t / 2\right\}},
$$

where $\omega_{j}$ is the frequency of the $j$-th mode, which is $\omega_{j}=\sin (\pi j / n) . \Delta t, f(n)$ and $v$ are determined from numerical simulations [15]. However we can also estimate these values by solving the collision of the chain with $n=2$ :

$$
\begin{aligned}
\Delta t & =\frac{\pi}{\sqrt{2} \omega}=2.22 / \omega \quad(2.31 / \omega), \\
f(n) & \simeq \frac{2 \sqrt{2}}{\pi} n=0.90 n \quad(0.867 n), \\
v & =\frac{\pi}{2 \sqrt{2}} v_{0}=1.11 v_{0} \quad\left(1.15 v_{0}\right),
\end{aligned}
$$

where the values in brackets were obtained from a numerical simulation of an $n=500$ chain. We can estimate an approximate value of $\eta$ by using Eqs. (8), (9) and (10). 


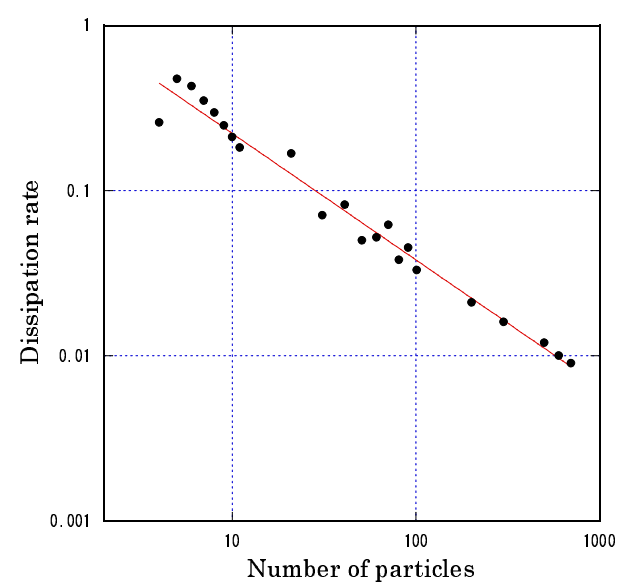

FIG. 3: A log-log plot of dissipation rate versus number of particles. The solid line has slope $-2 / 3$. (Reduced units where $c_{l}=\tau=1$.)

To obtain the asymptotic behavior of $1-\eta$ for large $n$, we expand the dispersion relation.

$$
\omega_{j}=\sqrt{k / m} \frac{\pi j}{n}\left(1-\frac{\pi^{2} j^{2}}{24 n^{2}}+\cdots\right)
$$

Substituting only the leading order of Eq.(11) into Eq.(何), we have $\eta=1$. Taking the second order into account, we obtain

$$
1-\eta \sim 0.652 n^{-\frac{2}{3}},
$$

in the limit $n \rightarrow \infty$. This relationship agrees very well with the numerical result. In Fig. 3, we plot the dissipation rate $1-\eta$ versus $n$.

\section{UNHARMONIC CHAINS}

In the case of harmonic chains, COR and contact time were independent of initial velocity $v_{0}$. Here, we introduce nonlinearity to the springs in the chain (model B).

In order to maintain a universal viewpoint, we first consider a velocity scale characterized by the nonlinearity of the spring. Let $U(x)$ be the potential energy of the spring, which is chosen to be $U(1)=0$ assuming the natural length of the spring to be unity. We can define the amplitude $x^{*}$ at which the harmonic term and the sum of the remaining terms are equal in the Taylor expansion, i.e., $x^{*}$ is given by the solution of

$\left.\frac{1}{2} \frac{d^{2} U(x)}{d x^{2}}\right|_{x=1}\left(x^{*}-1\right)^{2}=U(x)-\left.\frac{1}{2} \frac{d^{2} U(x)}{d x^{2}}\right|_{x=1}\left(x^{*}-1\right)^{2}$.

The velocity which corresponds to $x^{*}$ is

$$
v^{*}=\sqrt{2 U\left(x^{*}\right)} .
$$

Hereafter, we discuss the velocity dependence of the collisions on the scale of $v^{*}$.

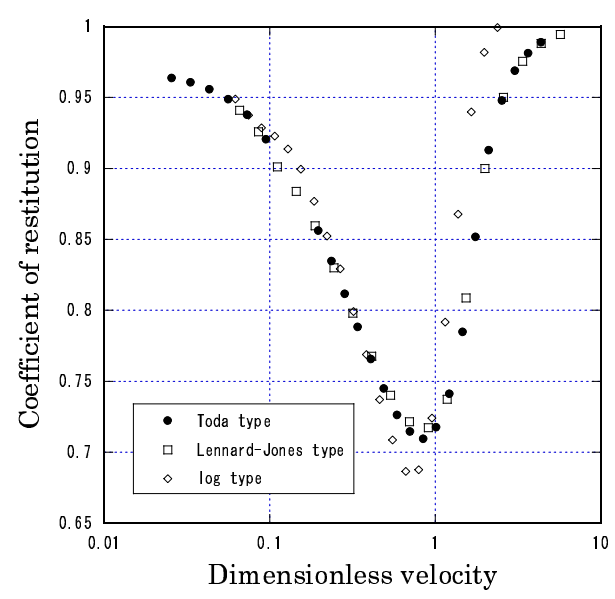

FIG. 4: Coefficient of restitution for the collisions of log-, Toda- and Lennard-Jones-type chains as a function of collision velocity. The chains consist of 100 particles.

Let us choose the following three types of potential and compare the results of simulation.

(a) Lennard-Jones potential

$$
U_{L J}(x)=\frac{1}{72}\left\{\left(\frac{1}{x}\right)^{12}-2\left(\frac{1}{x}\right)^{6}\right\}
$$

(b) Toda potential

$$
U_{\text {toda }}(x)=\frac{a}{b} e^{-b(x-1)}+a(x-1)
$$

(c) Log-type potential

$$
U_{\log }(x)=x-\log (x)
$$

For the Toda potential, we set $a b=1$ and $b=10$. Each potential has one minimum at $x=1$ and the function forms are similar. However, increasing behaviors of the repulsive forces derived from the three potentials are, in a very short distance, different from one another. In Fig. 4, we plot the COR versus initial velocity for each case, as determined by numerical simulations. In the limit of small velocity, it is clear that COR approaches the value obtained for the harmonic chain. The COR decreases with increasing initial velocity and has a minimum near $v=1$. We note that the COR lies on almost the same curve for all three types of potential.

Figure 5 shows $n$-dependence of $\eta$ for different values of initial velocity. In very low-velocity collision, as expected from the result for model $\mathrm{A}$, dissipation rate $1-\eta$ will approach to zero in the thermodynamic limit. Near $v_{0}=1$, the COR does not approach unity but remains a constant less than unity even in the thermodynamic limit. We can hence conclude that nonlinearity of the potential $U(x)$ causes the dissipation, which does not appear in model A.

Using a technique based on the perturbative theory, we consider the collision of model B for small initial incident 


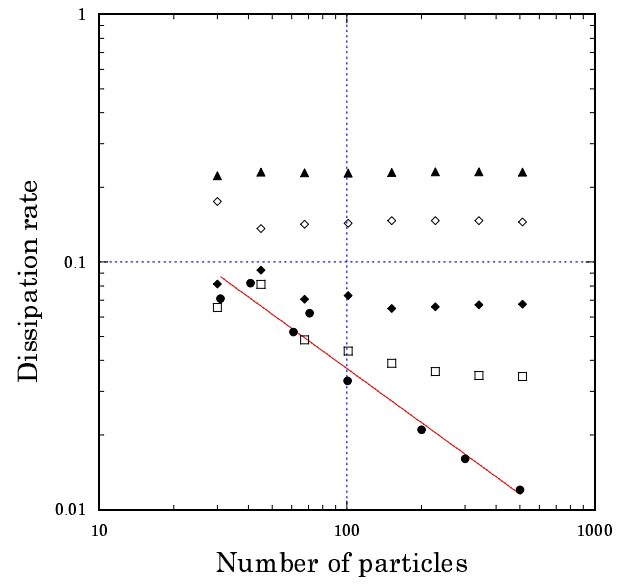

FIG. 5: The relationship between dissipation rate and number of particles in the collision of Lennard-Jones-type chain. Each plot corresponds to a different initial velocity: $v_{0}=0.780(\boldsymbol{\Lambda})$, $0.390(\diamond), 0.160(\diamond), 0.078(\square)$ and $0.008(\bullet)$.

velocity $v_{0} \ll 1$. During collision processes, the particle $j=1$ transmits vibration force to its neighbor particle. Let us regard this force as external force $F(t)$ which acts on the chain. The characteristic frequency of this force is $\omega_{\text {ext }}=2 \pi / \Delta t \simeq 2 \sqrt{2} \omega$. This frequency is higher than any frequency of fundamental modes of the chain. Hence, no fundamental mode is excited by the force. In this situation, the amplitude of each particle's vibration is rapidly damped progressively into the chain, i.e., the particle $j=1$ has the largest amplitude during collision. It is expected that $\Delta t$ will shorten with increasing initial velocity. As a first approximation, we take into account only the change of $\triangle t$ against initial velocity $v_{0}$ in Eq. (7). To estimate the velocity dependence of $\Delta t$, let us consider the collision of a Lennard-Jones-type chain with $n=2$. In this case, particle $j=1$ collides with the wall two times. The Hamiltonian is

$$
\mathcal{H}=\frac{m}{2}\left(\dot{x}_{1}^{2}+\dot{x}_{2}^{2}\right)+U_{L J}\left(x_{2}-x_{1}-1\right)+V_{w} .
$$

Let $x=x_{2}-x_{1}-\ell$ and $x_{q}=\left(x_{2}+x_{1}\right) / 2$. For initial conditions, we adopt Eq. (6). Immediately after the first collision of particle $x_{1}, x$ and $x_{g}$ and their time derivatives become

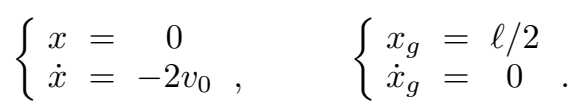

Taking into account the second order of $U_{L J}$ and solving the equation of motion with the initial conditions of Eq. (14) in the first-order perturbation theory, one can obtain the interval $\Delta t$ between first and second collisions of particle $x_{1}$ as

$$
\triangle t \simeq \frac{\pi}{\sqrt{2}}\left(1-\alpha \frac{v_{0}}{v^{*}}\right)
$$

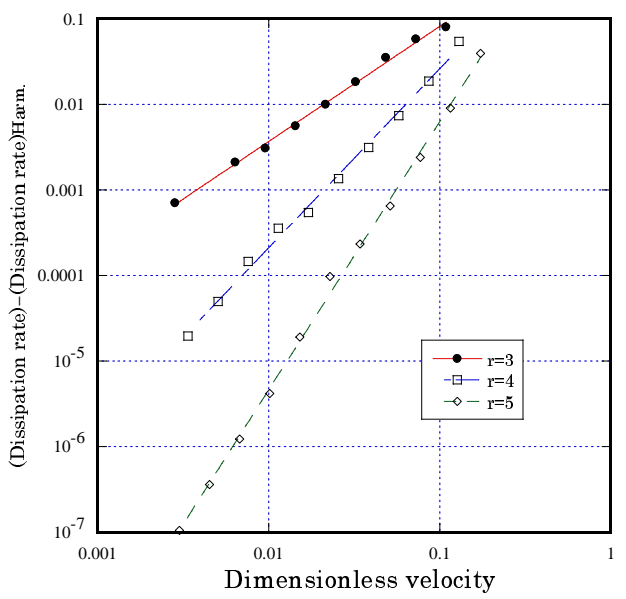

FIG. 6: Increase of dissipation rates. We plot the dissipation rate of model $\mathrm{B}$ minus that of model A for $r=3,4$ and 5 . Fitted lines have slopes 1.34, 2.09 and 3.14, respectively.

with $\alpha=0.808$. Substituting this into Eq. (7), we have

$$
E_{j}=\frac{4}{n} \cos ^{2}\left(\frac{\pi j}{2 n}\right) \frac{\sin ^{2}\left[\frac{1}{2} \omega_{j} f(n) \triangle t(1-\alpha \tilde{v})\right]}{\sin ^{2}\left[\frac{1}{2} \omega_{j} \triangle t(1-\alpha \tilde{v})\right]} .
$$

In the limit $n \rightarrow \infty, \omega_{j}$ can be replaced with the linear form

$$
\omega_{j}=\omega \frac{\pi j}{n} .
$$

When $v / c_{l} \ll 1$, Eq. (16) can be approximated as

$$
E_{j} \simeq \frac{32}{n \pi^{2}}\left(\frac{n}{j}\right)^{2} \sin ^{2}\left[\alpha v \pi n\left(\frac{j}{n}\right)\right] .
$$

Substituting this into Eq. (3), we have the dissipation rate

$$
\begin{aligned}
1-\eta=\sum_{j=1}^{n} \frac{E_{j}}{E} & \simeq \frac{64}{n \pi^{4}} \int_{0}^{1} d x \frac{1}{x^{2}} \sin ^{2}(\alpha v n x) \\
& \simeq \frac{64}{\pi^{3}} \alpha v \int_{0}^{\infty} d x \frac{\sin ^{2}(\alpha x)}{x^{2}} \\
& =C v,
\end{aligned}
$$

where $C=\frac{32}{\pi^{2}} \alpha \simeq 2.61$. This implies that the dissipation rate $1-\eta$ increases with the power law $v^{p}$ where $p=1$. This result agrees with numerical simulation for low-velocity collision. However the constant $C$ is not in accord with the above result (our numerical simulation gives $C=0.66$ ).

The above result directly depends on the exponent of the lowest un-harmonic term of interparticle potential energy. In the case of Lennard-Jones potential, the exponent $r=3$. The exponent is the same for other types of chains. Let us discuss more general cases. Suppose that the interparticle potential energy can be expanded around its equilibrium position as

$$
U(x)=\frac{1}{2} x^{2}+c x^{r}+\text { higher order, }
$$




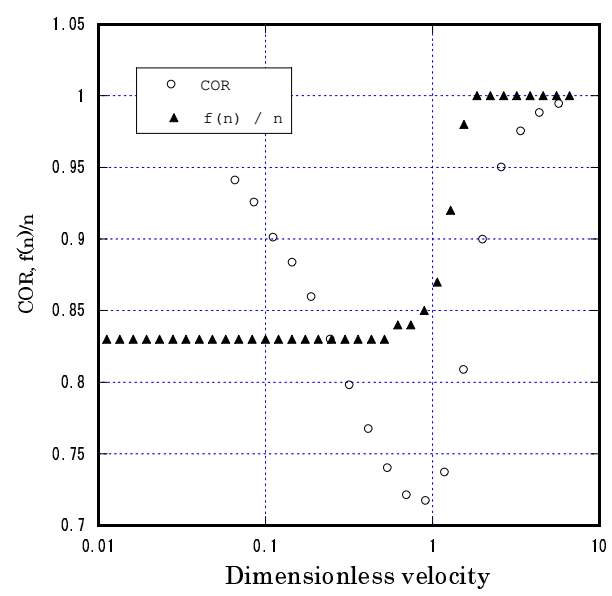

FIG. 7: Plot of ratio of $f(n)$ to $n$ and COR versus velocity for Lennard-Jones-type chain. Both increase at $\tilde{v} \approx 1$.

where the constant $c$ is a positive (negative) number when $r$ is even (odd). In this case, the contact time shortens as $v_{0}^{r-2}$. The dissipation rate hence increases as

$$
1-\eta \propto\left(\frac{v}{v^{*}}\right)^{r-2} .
$$

Taking potential energy Eq.(19) for the interaction of particles in model B, we plot numerical results of COR in Fig. 6 for $v \ll 1$. The results agree with Eq. (20).

When the particles in the chain can be regarded as rigid spheres, interparticle interaction is of the $\delta$ function type. Collision between the two spheres is reduced to a simple exchange of their momenta. We can exactly solve the entire dynamics of the collision between the chain and a rigid wall. The sphere $j=1$ collides with the wall $n$ times and no internal vibration remains after the collision, i.e., the COR is exactly unity in this case.

In the limit of high-velocity collision of model B, particles interact like rigid spheres because $U_{L J}, U_{\text {toda }}$ and $U_{l o g}$ all behave like hard core potentials within a very short distance. In Fig. 7, we show the COR of a LennardJones-type chain and $f(n) / n$ versus collision velocity on the same plot. The rate $f(n) / n$ approaches unity for $v>1$. This indicates that particles interact like rigid spheres in high-velocity collision. Consequently COR increases in the high-velocity regime. This is a feature which only the one-dimensional model exhibits and is unrealistic. In real systems, plastic deformation is crucial in such high-velocity collisions.

\section{SUMMARY}

We have presented a simple one-dimensional microscopic model of colliding bodies to understand the energy dissipation process. Lennard-Jones, Toda and Log-type potentials are chosen as interactions between particles. We found that the COR depends on the initial velocity and is minimum at $v / v * \simeq 1$. These behaviors are independent of the potential form. In low-velocity collisions, the relationship between the energy dissipation rate and collision velocity is derived using perturbation methods.
[1] L.D. Landau, and E.M. Lifshitz, Theory of elasticity (2nd ed.). Pergamon Press. (1960)

[2] S.P. Timoshenko and J.N. Goodier, Theory of elasticity (3rd ed.). McGraw-Hill (1970)

[3] H. Hertz, J. Reine Angew Math. 92,156 (1882)

[4] K.L. Johnson, Contact Mechanics. Cambridge University Press. (1985)

[5] G. Kuwabara, and K. Kono, Jpn. J. Appl. Phys. 26, 1230 (1987)

[6] T. Schwager and T. Pöschel, Phys. Rev. E 57, 650 (1998)

[7] R. Ramirez, T. Pöschel, N.V. Brilliantov, and T. Schwager, Phys. Rev. E 60, 4465 (1999)

[8] C.V. Raman, Phys. Rev. 12, 442 (1918)

[9] R. Sondergaard, K. Chaney, and C.E. Brennen, J. Appl. Mech. 57, 694 (1990)
[10] L. Labous, A.D. Rosato, and R.N. Dave, Phys.Rev.E 56, 5717 (1997)

[11] G. Giese and A. Zippelius, Phys. Rev. E 54, 4828 (1999)

[12] F. Gerl and A. Zippelius, Phys. Rev. E 59, 2361 (1999)

[13] H. Hayakawa and H. Kuninaka, Proc. 9th. Nisshin Engineering Particle Thchnology Int. Symp., Kyoto, 2001, p.82 (cond-mat/0106145)

[14] M. Sugiyama and N. Sasaki, J. Phys. Soc. Jpn. 68, 1859 (1999)

[15] A.G. Basile, and R.S. Dumont, Phys. Rev. E 61, 2015 (2000)

[16] J.A. Zukas, T. Nicholas, H.F. Swift, L.B. Greszczuk and D.R. Curran, Impact Dynamics. Krieger Publishing Company. (1992) 